\title{
Microcrack connectivity in rocks: a real-space renormalization group approach for 3D anisotropic bond percolation
}

\section{Ya-Pu Zhao, Jiangce Chen, Quanzi Yuan and Chemin Cheng}

State Key Laboratory of Nonlinear Mechanics (LNM), Institute of Mechanics,

Chinese Academy of Sciences, Beijing 100190, People's Republic of China

E-mail: yzhao@imech.ac.cn

Received 25 August 2015

Accepted for publication 27 October 2015

Published 28 January 2016

Online at stacks.iop.org/JSTAT/2016/013205

doi:10.1088/1742-5468/2016/01/013205

\begin{abstract}
The onset of microcrack conductivity in anisotropic rocks, which is a concern in hydraulic fracturing, was treated as a three-dimensional (3D) bond percolation problem where the bonds in the three orthogonal directions are presented with different probabilities. A real-space renormalization group (RNG) approach was expanded to calculate the critical behavior in this percolation problem, which was demonstrated to have improved accuracy for estimating critical points. A 3D phase diagram for the anisotropic percolation problem was obtained to present the relation between rock permeability and crack density, which is suitable for the needs of engineering for its accuracy and visualization.
\end{abstract}

Keywords: fracking, percolation, permeability, anisotropy, renormalization group 


\section{Contents}

1. Introduction 2

2. Methodology 4

3. Models 4

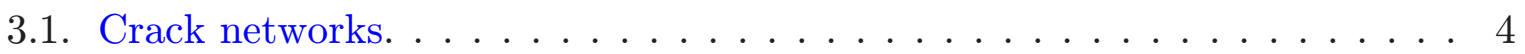

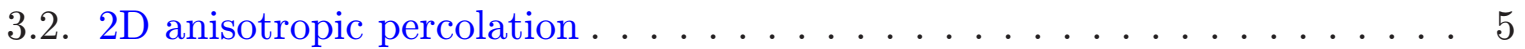

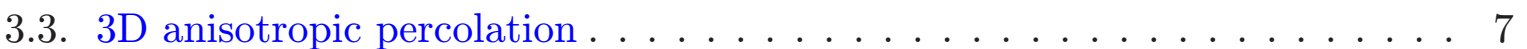

4. Results and discussion 9

5. Conclusions 15

$\begin{array}{ll}\text { Appendix A } & 16\end{array}$

$\begin{array}{ll}\text { Appendix B } & 16\end{array}$

$\begin{array}{ll}\text { Acknowledgments } & 17\end{array}$

$\begin{array}{ll}\text { References } & 17\end{array}$

\section{Introduction}

Hydraulic fracturing, also known as fracking, is the process of drilling and injecting fluid into the deep ground at a high pressure to enhance the permeability in petroleum and gas reservoirs [1]. It still remains a mystery how to explain the topology, geometry, and evolution of the crack system in fracking [2]. An understanding of the underlying mechanisms responsible for fracture evolution is necessary for accurate predictions of fracture flow attributes [3]. It seems that analysis based on the crack system might bring out significant estimation and prediction for the permeability of shale, both prefractured and after-fractured.

Shale is a fine-grained rock and its granular permeability is extremely low. Unfractured shale is often considered impermeable. Joints, fractures, and faults provide the flow paths necessary for shale 'permeability'. The connectivity of these fractures, called microcrack connectivity, is what determines fluid conductivity and permeability. These fractures are thought to play a significant role in the fracking process [4] . Microcrack connectivity is not equal to fluid connectivity because when the size of the fractures is lower than a certain value, it would be too narrow for fluid to pass through, unless only the fractures suitable for fluid to flow through are considered in the model. Therefore, the scale of the relevant fractures is thought to be determined according to the specific fluid whose permeability in shale is the concern.

Percolation theory was originally introduced to study fluid connectivity in porous media which are considered as a kind of network [5]. The core of percolation is the study of 
connectivity [6]. A real-space renormalization group approach (RNG) was applied for twodimensional (2D) bond percolation using a scaling transformation in a square lattice and triangular lattice [7], which is the first time that the RNG is applied in percolation. The basic idea of the RNG is to connect the parameter distribution with the state of the network by scaling transformation. Harris and his colleagues [8] formulated an RNG through the relation between the $s$-state Ashkin-Teller-Potts (ATP) model and the percolation given by Kasteleyn and Fortuin [9]. Stinchcombe and Watson [10] generalized their own work to deal with the percolation conductivity of dilute resistor networks and obtained the spinwave property in dilute ferromagnets. Reynolds et al [11] expanded this method to 2D site percolation and studied the inclusion of a 'ghost spin' in 1D through choosing a subsection made of two bonds and two sites to make possible the calculation of remaining exponents. To study microcrack connectivity in rocks, Madden [12] restricted the anisotropy of his models to transversely isotropic symmetry and obtained a rock connectivity phase diagram for a transversely isotropic crack distribution. Additionally, he introduced the interaction between different length scales, which improved the results of equivalent media theory.

Differing from the standard one, there is another kind of percolation theory. Instigated by the issue of one fluid displacing another from a porous medium under the effect of capillary forces, invasion percolation (IP), as a new kind of percolation, was introduced [13]. In this model, the flow of two immiscible fluids-an invading fluid, such as water, and a defending fluid, such as oil-is studied. The pores that are occupied by the defending fluid are simplified as sites in a $2 \mathrm{D}$ square grid. The fluid would flow through the bonds which connect the sites. An invading fluid is injected in a site and regarded as a point source. It chooses the weakest bonds as the pathway to displace the defending fluid at each time step. Furuberg and his co-workers [14] studied the dynamics of displacement fronts in IP and obtained the probability of invading a site at a distance $r$ from a reference site at a time $t$ after that site was invaded, which is related to the fractal dimension of the invaded region. From then on, based on IP, algorithms for a broad class of self-organized critical models were developed [15-19].

However, all those IP models have not solved the percolation threshold in 3D anisotropic percolation thoroughly. The results obtained from IP are based on the Monte Carlo method, which means repeating random invasion processes with specific parameters. This method has not derived general relations between the crack density and the system connectivity. Nakanishi et al [20] developed a 3D bond percolation model with anisotropic bond occupation probabilities in order to study the crossover behavior of the effective spatial dimension of the system. In terms of describing critical behavior, the results of this model are far from satisfactory. The isotropic percolation threshold $p_{c}$ obtained from Monte Carlo simulations on a simple cubic lattice should be about 0.25 [21], while, in Nakanishi et al's model, $p_{c}$ is calculated to be about 0.0477 , as shown in appendix A. So, we modified the 3D anisotropic bond percolation model. The description of critical behavior has progressed and $p_{c}$ is calculated to be about 0.2155 . We drew a 3D anisotropic phase diagram to indicate whether a path of connected cracks exits by passing through the rock according to the crack density. It presents relations between permeability and rock density visually and may provide significant help for rock permeability-related engineering, such as hydraulic fracturing. A 2D anisotropic bond percolation will be dealt with firstly to introduce the formal structure of the RNG approach, and then an RNG approach for 3D anisotropic bond percolation will be presented. 


\section{Methodology}

The RNG approach is an approximation method but it can expose the fundamental behaviors of a system in a compact way, which is very useful for modeling large complex systems. A large system is divided into small similar subsections to investigate their properties. Each whole subsection is then considered as an element of a largerscale subsection, which is called renormalization. The properties of this larger-scale subsection are then determined, and the subsection is treated as an element of an even larger-scale subsection, and the whole process is repeated again [12]. All these renormalizing operations are combined to the renormalization group. In the parameter space, there are certain points called fixed points where no change takes place during the scaling procedure. The fixed points can be divided into two kinds, stable fixed points and unstable fixed points. Stable fixed points, which attract another near them as it moves up in scale, represent the stable state of the system. Unstable fixed points, which are like peaks and saddle points in a topographic map, represent the critical state of the system. In 3D anisotropic percolation, surfaces consisting of fixed points separate the parameter space into several regions which represent a stable state of the system.

The scaling procedure or renormalizing operation can be described as iteration functions in mathematics which are denoted as $R(\mu)$ where $\mu$ is the parameter space of the system. Appendix B contains a renormalizing operation of $2 \mathrm{D}$ isotropic percolation as an instance.

After every renormalizing operation, the resolution about the positions of the bonds decreases, which obscures the information about the shape of the connecting paths while clearing the probability of the existence of the connecting paths. As the renormalizing operation cycles infinitely, the information about the connecting paths' shape totally vanishes, while it is determined whether the connecting paths through the $X, Y$ and $Z$ directions, respectively, exist. The information property is consistent during the process. A bond network is randomly created based on the occupying probability of the bonds in the $X, Y$ and $Z$ directions, the anisotropy of the bond distribution. Because it is a random process, the geomorphic information of the bond network is not given, which is consistent with the disappearance of the information about the shape of the connecting paths when the renormalizing operation cycles infinitely. The renormalizing operation transmutes the anisotropy of the network from the occupying probabilities of the bonds in the $X, Y$ and $Z$ directions, finally to the existing probabilities of the connecting paths through the $X, Y$ and $Z$ directions.

\section{Models}

\subsection{Crack networks}

The rock was transformed into ae network by several simplifications. First, the cracks were considered as lines because the length of a crack is larger than its width by about two orders of magnitude. Actually, microcracks are typically a few micrometers to a millimeter in length and $0.01 \mu \mathrm{m}$ to a few micrometers in width [12]. Besides, the 
distinction between holes and cracks was neglected. Connections in rocks include holes and cracks; however, both of them can be treated as the probabilities of the nearest connection in rocks. And it is supposed that the lengths of the cracks are in the same scale. Madden [12] considered the interaction between different length scales by incorporating the addition of cracks whose lengths are appropriate for the current scale in every scaling procedure. It will make the results more accurate but lose the universality of the theory for the distributions of the crack lengths vary from rock to rock. In order to get universal results for 3D anisotropic percolation, the different length scales were not taken into account.

Then, crack density was defined as the probability of a bond presenting in a certain position. The parameters that can be observed are the numbers, lengths, and orientations of cracks on surfaces. If the cracks observed on a surface were split into two groups, each group having normal lines oriented within $45^{\circ}$ of one of the two principal directions, $X$ and $Y$, the density parameters are $N_{x} L^{2} / S$ and $N_{y} L^{2} / S$ which are the crack number per unit area. $N_{x}$ and $N_{y}$ are the numbers of cracks in the $X$ direction and $Y$ direction, respectively. $L$ is the crack length and $S$ is the area of the surface observed [12] . $N_{z} L^{2} / S$ is defined in the same way. Hence, the rock model is obtained in the shape of a network.

\subsection{D anisotropic percolation}

The 2D anisotropic lattice, as shown in figure 1(a), consists of subsections that both cover the lattice and maintain its symmetry. Each $X$ and $Y$ bond of a regular lattice can be present, with probability $p_{x}$ and $p_{y}$, respectively, or absent, with probability $1-p_{x}$ and $1-p_{y}$. According to the definition above, $p_{x}$ and $p_{y}$ can be expressed as

$$
p_{x}=\frac{N_{x} L^{2}}{S}, p_{y}=\frac{N_{y} L^{2}}{S} .
$$

An illustration of the $2 \mathrm{D}$ scaling procedure is provided. As shown in figure 1(a), through transforming the subsections into elements, the square lattice is scaled by a factor of $b=2$ which is the change of length after scaling. The probabilities $p_{x}$ and $p_{y}$ of a nearest neighbor connection in the $X$ and $Y$ directions on the original lattice scales are combined into the probabilities $p_{x}^{\prime}$ and $p_{y}^{\prime}$ for a connection between the nearest neighbors on the new lattice. $p_{x}^{\prime}$ and $p_{y}^{\prime}$ are determined by

$$
p_{x}^{\prime}=R_{x}\left(p_{x}, p_{y}\right), p_{y}^{\prime}=R_{y}\left(p_{x}, p_{y}\right) .
$$

The probability of a nearest neighbor connection in the $X$ or $Y$ direction on the new lattice depends on the combination of the paths on the original subsection. For example, if the original subsection 'got across' in the $X$ direction, there would be an $X$ bond on the new lattice, as shown in figure 1(a). And all the probabilities of the possible combinations in the subsections were summed up to obtain $R_{x}\left(p_{x}, p_{y}\right)$ and $R_{y}\left(p_{x}, p_{y}\right)$.

All the possible combinations of the paths and their probabilities are shown in figure 1(b). The probabilities of a nearest neighbor connection in the $X$ and $Y$ directions on the new lattice are 


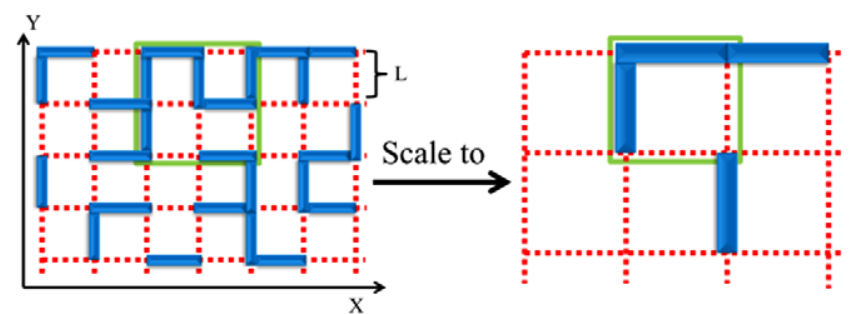

(a)

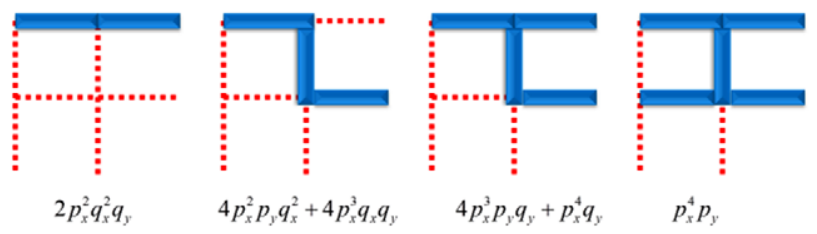

(b)

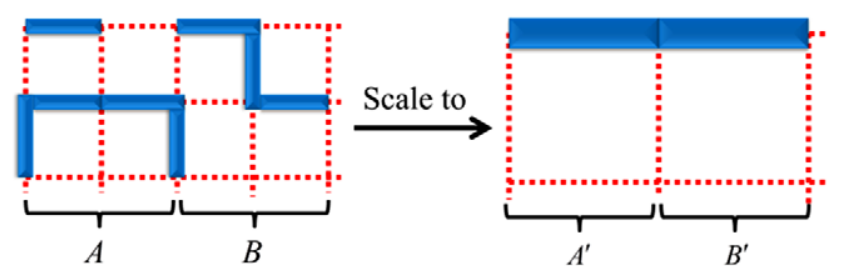

(c)

Figure 1. A sample of crack distribution in a 2D lattice. The red dotted lines are the unoccupied positions and the blue solid lines are the bonds. (a) The square lattice is scaled into a new lattice by a factor $b=2$. (b) All possible combinations of the paths and their probabilities. (c) An illustration of the 'surface effect'. Two disconnected subsections, $A$ and $B$, become two connected elements, $A^{\prime}$ and $B^{\prime}$, after scaling.

$$
\left\{\begin{array}{l}
p_{x}^{\prime}=2 p_{x}^{2} q_{x}^{2} q_{y}+4 p_{x}^{2} p_{y} q_{x}^{2}+4 p_{x}^{3} q_{x} q_{y}+4 p_{x}^{3} p_{y} q_{x}+p_{x}^{4} q_{y}+p_{x}^{4} p_{y} \\
p_{y}^{\prime}=2 p_{y}^{2} q_{y}^{2} q_{x}+4 p_{y}^{2} p_{x} q_{y}^{2}+4 p_{y}^{3} q_{y} q_{x}+4 p_{y}^{3} p_{x} q_{y}+p_{y}^{4} q_{x}+p_{y}^{4} p_{x}
\end{array}\right.
$$

They have the form of a scaling relation $\mu^{\prime}=R(\mu)$, where $\mu$ is the parameter space of the system. Here $\mu$ is just the probabilities $p_{x}$ and $p_{y}$. The fixed point $\mu^{*}$ for the transformation is then determined by

$$
\mu^{*}=R\left(\mu^{*}\right)
$$

and, in its neighborhood, the transformation can be linearized to

$$
\delta \mu^{\prime}=R^{L}(\delta \mu)
$$

where $\delta \mu=\mu-\mu^{*}$. If $R^{L}$ has eigenvalues $\lambda_{i}$ with $\lambda_{1}>1>\lambda_{2} \ldots$, the path to the critical surface is determined by $\lambda_{1}$. As for anisotropic percolation where $p_{x}=p_{y}=p$ and $R_{x}\left(p_{x}, p_{y}\right)=R_{y}\left(p_{x}, p_{y}\right)=R(p), \quad \lambda_{1}=\partial R /\left.\partial p\right|_{p=p_{c}}$. The correlation-length index $v$ is related to $\lambda_{1}$ by 
Microcrack connectivity in rocks

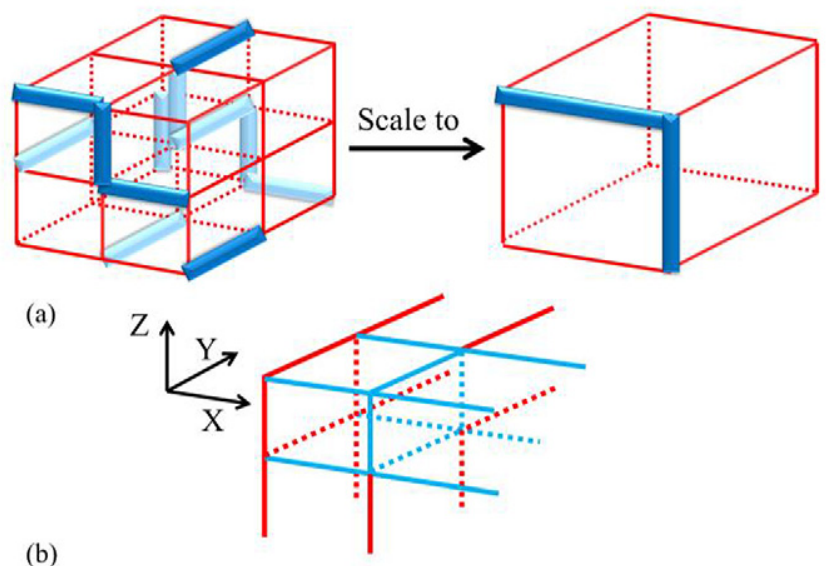

Figure 2. An illustration of the $3 \mathrm{D}$ scaling procedure. (a) Scale the cubic lattice into a new lattice by a factor $b=2$. (b) The $2 \times 2 \times 2$ formation cubic subsection. The blue lines are the positions of the bonds that affect the connection in the $X$ direction which include eight $X$ bonds, four $Y$ bonds and four $Z$ bonds.

$$
b^{1 / v}=\lambda_{1}
$$

The mean size of these (finite) clusters diverges as $p$ approaches $p_{c}$, and for $p>p_{c}$ there is a non-zero probability that a chosen bond will belong to an infinite cluster [10]. An associated correlation length $\xi$ which diverges at $p_{c}$ can be defined as

$$
\xi \propto\left(p-p_{c}\right)^{-v}
$$

\subsection{D anisotropic percolation}

Similar to the definitions in $2 \mathrm{D}, p_{x}, p_{y}$ and $p_{z}$ in $3 \mathrm{D}$ can be expressed as

$$
p_{x}=\frac{N_{x} L^{2}}{S}, p_{y}=\frac{N_{y} L^{2}}{S}, p_{z}=\frac{N_{z} L^{2}}{S} .
$$

An illustration of the 3D scaling procedure is provided. As shown in figure 2(a), through transforming the subsections into elements, the cubic lattice is scaled by a factor of $b=2$ which is the ratio of the length change after-scaling. The probabilities $p_{x}$, $p_{y}$ and $p_{z}$ of a nearest neighbor connection in the $X, Y$ and $Z$ directions on the original lattice scales are combined into the probabilities $p_{x}^{\prime}, p_{y}^{\prime}$ and $p_{z}^{\prime}$ for a connection between the nearest neighbors on the new lattice. $p_{x}^{\prime}, p_{y}^{\prime}$ and $p_{z}^{\prime}$ are determined by

$$
p_{x}^{\prime}=R_{x}\left(p_{x}, p_{y}, p_{z}\right), p_{y}^{\prime}=R_{y}\left(p_{x}, p_{y}, p_{z}\right), p_{z}^{\prime}=R_{z}\left(p_{x}, p_{y}, p_{z}\right)
$$

The probability of a nearest neighbor connection in the $X$ direction on the new lattice is taken as an example. The positions that affect the connection in the $X$ direction are shown in figure 2(b). There are eight $X$ bonds, two $Y$ bonds and two $Z$ bonds. Obviously, to make the combination 'get across' in the $X$ direction, there need to be two bonds at least and 12 bonds at most. All the possible combinations of the paths were divided into 11 types by the number of the bonds, and then all the probabilities 
of the combinations of the paths are summed up to obtain the probability of a nearest neighbor connection in the $X$ direction on the new lattice. So

$$
p_{z x}^{\prime}=R_{x}\left(p_{x}, p_{y}, p_{z}\right)=R_{x}^{2 \mathrm{bonds}}+R_{x}^{3 \mathrm{bonds}}+\cdots+R_{x}^{12 \mathrm{bonds}}
$$

where $R_{x}$ with different bonds are expressed, respectively, as follows:

$$
\begin{aligned}
& R_{x}^{2 \text { bonds }}=4 p_{x}^{2} q_{x}^{6} q_{y}^{2} q_{z}^{2}, \\
& R_{x}^{3 \mathrm{bonds}}=24 p_{x}^{3} q_{x}^{5} q_{y}^{2} q_{z}^{2}+12 p_{x}^{2} q_{x}^{6} p_{y} q_{y} q_{z}^{2}+12 p_{x}^{2} q_{x}^{6} q_{y}^{2} p_{z} q_{z} \\
& R_{x}^{4 \text { bonds }}=60 p_{x}^{4} q_{x}^{4} q_{y}^{2} q_{z}^{2}+56 p_{x}^{3} q_{x}^{5} p_{y} q_{y} q_{z}^{2}+56 p_{x}^{3} q_{x}^{5} q_{y}^{2} p_{z} q_{z}+12 p_{x}^{2} q_{x}^{6} p_{y}^{2} q_{z}^{2} \\
& +12 p_{x}^{2} q_{x}^{6} q_{y}^{2} p_{z}^{2}+32 p_{x}^{2} q_{x}^{6} p_{y} q_{y} p_{z} q_{z} \\
& R_{x}^{\text {5bonds }}=56 p_{x}^{5} q_{x}^{3} q_{y}^{2} q_{z}^{2}+136 p_{x}^{4} q_{x}^{4} p_{y} q_{y} q_{z}^{2}+136 p_{x}^{4} q_{x}^{4} q_{y}^{2} p_{z} q_{z}+40 p_{x}^{3} q_{x}^{5} p_{y}^{2} q_{z}^{2} \\
& +40 p_{x}^{3} q_{x}^{5} q_{y}^{2} p_{z}^{2}+120 p_{x}^{3} q_{x}^{5} p_{y} q_{y} p_{z} q_{z}+32 p_{x}^{2} q_{x}^{6} p_{y}^{2} p_{z} q_{z}+32 p_{x}^{2} q_{x}^{6} p_{y} q_{y} p_{z}^{2} \text {, } \\
& R_{x}^{6 \mathrm{bonds}}=28 p_{x}^{6} q_{x}^{2} q_{y}^{2} q_{z}^{2}+112 p_{x}^{5} q_{x}^{3} p_{y} q_{y} q_{z}^{2}+112 p_{x}^{5} q_{x}^{3} q_{y}^{2} p_{z} q_{z}+64 p_{x}^{4} q_{x}^{4} p_{y}^{2} q_{z}^{2}+64 p_{x}^{4} q_{x}^{4} q_{y}^{2} p_{y}^{2} \\
& +244 p_{x}^{4} q_{x}^{4} p_{y} q_{y} p_{z} q_{z}+72 p_{x}^{3} q_{x}^{5} p_{y}^{2} p_{z} q_{z}+72 p_{x}^{3} q_{x}^{5} p_{y} q_{y} p_{z}^{2}+16 p_{x}^{2} q_{x}^{6} p_{y}^{2} p_{z}^{2}, \\
& R_{x}^{7 \mathrm{bonds}}=8 p_{x}^{7} q_{x} q_{y}^{2} q_{z}^{2}+56 p_{x}^{6} q_{x}^{2} p_{y} q_{y} q_{z}^{2}+56 p_{x}^{6} q_{x}^{2} q_{y}^{2} p_{z} q_{z}+56 p_{x}^{5} q_{x}^{3} p_{y}^{2} q_{z}^{2}+56 p_{x}^{5} q_{x}^{3} q_{y}^{2} p_{z}^{2} \\
& +224 p_{x}^{5} q_{x}^{3} p_{y} q_{y} p_{z} q_{z}+128 p_{x}^{4} q_{x}^{4} p_{y}^{2} p_{z} q_{z}+128 p_{x}^{4} q_{x}^{4} p_{y} q_{y} p_{z}^{2}+48 p_{x}^{3} q_{x}^{5} p_{y}^{2} p_{z}^{2} \text {, } \\
& R_{x}^{8 \mathrm{bonds}}=p_{x}^{8} q_{y}^{2} q_{z}^{2}+16 p_{x}^{7} q_{x} p_{y} q_{y} q_{z}^{2}+16 p_{x}^{7} q_{x} q_{y}^{2} p_{z} q_{z}+28 p_{x}^{6} q_{x}^{2} p_{y}^{2} q_{z}^{2}+28 p_{x}^{6} q_{x}^{2} q_{y}^{2} p_{z}^{2} \\
& +112 p_{x}^{6} q_{x}^{2} p_{y} q_{y} p_{z} q_{z}+112 p_{x}^{5} q_{x}^{3} p_{y}^{2} p_{z} q_{z}+112 p_{x}^{5} q_{x}^{3} p_{y} q_{y} p_{z}^{2}+68 p_{x}^{4} q_{x}^{4} p_{y}^{2} p_{z}^{2}, \\
& R_{x}^{9 \mathrm{bonds}}=2 p_{x}^{8} p_{y} q_{y} q_{z}^{2}+2 p_{x}^{8} q_{y}^{2} p_{z} q_{z}+8 p_{x}^{7} q_{x} p_{y}^{2} q_{z}^{2}+8 p_{x}^{7} q_{x} q_{y}^{2} p_{z}^{2}+32 p_{x}^{7} q_{x} p_{y} q_{y} p_{z} q_{z} \\
& +56 p_{x}^{6} q_{x}^{2} p_{y}^{2} p_{z} q_{z}+56 p_{x}^{6} q_{x}^{2} p_{y} q_{y} p_{z}^{2}+56 p_{x}^{5} q_{x}^{3} p_{y}^{2} p_{z}^{2} \\
& R_{x}^{10 \mathrm{bonds}}=p_{x}^{8} p_{y}^{2} q_{z}^{2}+p_{x}^{8} q_{y}^{2} p_{z}^{2}+4 p_{x}^{8} p_{y} q_{y} p_{z} q_{z}+16 p_{x}^{7} q_{x} p_{y}^{2} p_{z} q_{z}+16 p_{x}^{7} q_{x} p_{y} q_{y} p_{z}^{2} \\
& +28 p_{x}^{6} q_{x}^{2} p_{y}^{2} p_{z}^{2} \\
& R_{x}^{11 \text { bonds }}=2 p_{x}^{8} p_{y}^{2} p_{z} q_{z}+2 p_{x}^{8} p_{y} q_{y} p_{z}^{2}+8 p_{x}^{7} q_{x} p_{y}^{2} p_{z}^{2} \text {, } \\
& R_{x}^{12 \text { bonds }}=p_{x}^{8} p_{y}^{2} p_{z}^{2},
\end{aligned}
$$

and the expressions of $p_{y}^{\prime}$ and $p_{z}^{\prime}$ are obtained by rotating the positions of $x, y, z$. As discussed above, fixed points are ones that take no change during the scaling procedure, so

$$
p_{x}=p_{x}^{\prime}, p_{y}=p_{y}^{\prime}, p_{z}=p_{z}^{\prime},
$$

and the surfaces of stable fixed points and unstable fixed points were obtained. 


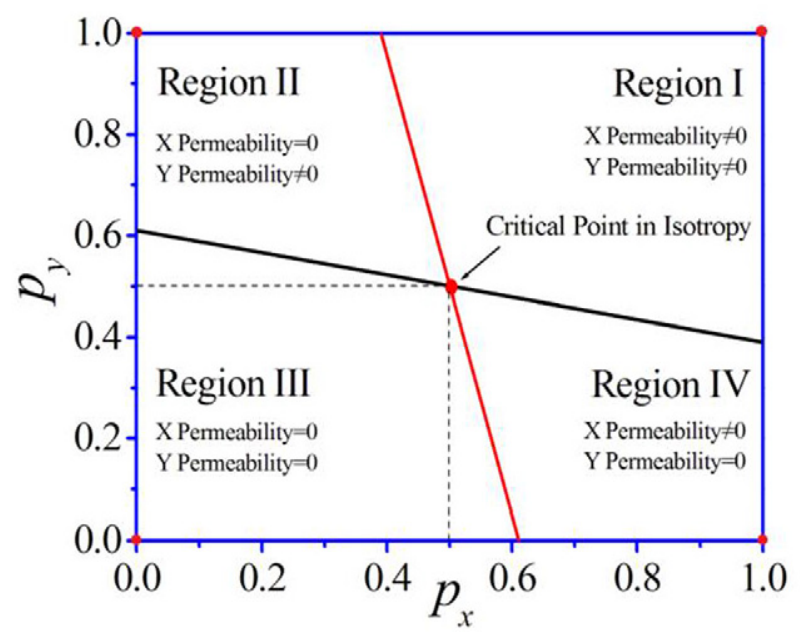

Figure 3. Rock connectivity phase for a $2 \mathrm{D}$ anisotropic crack distribution. The coordinates are the probabilities of the $X$ and $Y$ bonds being in a certain position. The red boundary is the curve, albeit seeming straight, of the upper function in equation (3) assuming $p_{x}=p_{x}^{\prime}$. The black boundary is the curve of the lower function in equation (3) assuming $p_{y}=p_{y}^{\prime}$. The points at $\left(p_{x}, p_{y}\right)=(0,0),(0,1)$, $(1,1)$ and $(1,0)$ are stable fixed points in isotropy representing four kinds of states. The fixed point at $(0.5,0.5)$ is a critical point in isotropy.

\section{Results and discussion}

For $2 \mathrm{D}$ isotropic percolation, $p_{x}=p_{y}$, setting $p_{x}^{\prime}=p_{x}$ and $p_{y}^{\prime}=p_{y}$, the fixed points were found at $p_{x}^{*}=0,1$ and $p_{y}^{*}=0,1$. An unstable fixed point gives $p_{c x}=p_{c y}=0.5$ (exact) and $v \approx 0.70$. According to the empirical formula

$$
p_{c}=\frac{2^{2-d} \cdot d}{z}
$$

where $z$ is the coordination number and $d$ is the dimensional number, which is found to approximate most known critical probabilities [12],$p_{c}=0.5$ in the square lattice where $z=4$ and $d=2$. The result is exactly the same as the empirical result.

In $2 \mathrm{D}$ anisotropic percolation, the results are presented in figure 3 which shows the fixed points and the phase boundaries between them. Equation (3), representing the renormalizing operation, is iterated until one of the fixed points is reached. Therefore, on the phase boundaries which consist of fixed points, the probabilities are unchanged after iteration, that is $p_{x}=p_{x}^{\prime}, p_{y}=p_{y}^{\prime}$. The red boundary is the curve of the upper function in equation (3) assuming $p_{x}=p_{x}^{\prime}$, though it seems straight. The black boundary is the curve of the lower function in equation (3) assuming $p_{y}=p_{y}^{\prime}$. The points at $\left(p_{x}, p_{y}\right)=(0,0),(0,1),(1,1)$ and $(1,0)$ are stable fixed points in isotropy representing four kinds of states. The fixed point at $(0.5,0.5)$ is a critical point in isotropy. Unstable 
Table 1. The approximations of the roots of equation (13).

\begin{tabular}{ll}
\hline Number & The approximations of the roots \\
\hline 1 & 0 \\
2 & 1 \\
3 & -0.277515182855068574438886349027541 \\
4 & 0.21550933014460972692369938507458 \\
5 & $-0.20527856991480574400245968325627-0.328252260333326647492034792644569 \mathrm{i}$ \\
6 & $-0.20527856991480574400245968325627+0.32825226033326647492034792644569 \mathrm{i}$ \\
7 & $0.63640848335856384603934383804721-0.62163638131289647119257855354781 \mathrm{i}$ \\
8 & $0.63640848335856384603934383804721+0.62163638131289647119257855354781 \mathrm{i}$ \\
9 & $1.4441731883777193566175935384599-0.62374835423894741380353016786315 \mathrm{i}$ \\
10 & $1.4441731883777193566175935384599+0.62374835423894741380353016786315 \mathrm{i}$ \\
11 & $0.9627173683934010878851219032092+0.26905311577229211571997917825455 \mathrm{i}$ \\
12 & $0.9627173683934010878851219032092-0.26905311577229211571997917825455 \mathrm{i}$ \\
\hline
\end{tabular}

fixed points compose the boundaries dividing the parameter space into four phases. Region I is the fully connected region. Region II has connections across the $Y$ direction but none in the $X$ direction. Region III is the fully disconnected region, and Region IV is disconnected in the $Y$ direction but connected in the $X$ direction.

For $3 \mathrm{D}$ isotropic percolation, $p_{x}=p_{y}=p_{z}$, setting $p_{x}^{\prime}=p_{x}, p_{y}^{\prime}=p_{y}$ and $p_{z}^{\prime}=p_{z}$, the fixed points are found at $p_{x}^{*}=0,1, p_{y}^{*}=0,1$ and $p_{z}^{*}=0,1$. The isotropic percolation threshold is one of the roots of the equation

$$
p=R_{x}(p, p, p)
$$

which is derived from equation (10) when $p_{x}=p_{y}=p_{z}=p$. The approximations of the roots of equation (13) are shown in table 1 . There is only one root that is a real number and between 0 and 1 , which is about 0.2155 . Therefore, $p_{c} \approx 0.2155$ and $v \approx 0.9651$, which are in good agreement with the result of Reynolds et al [11], $p_{c} \approx 0.22$ and $v \approx 1.04$, and the result of the work of Madden [12],$p_{c}=0.21$. The result is smaller than the one in $2 \mathrm{D}$ because there are more configurations in $3 \mathrm{D}$ which are the possible combinations of paths in the model when given the same crack density. In a cubic lattice where the coordination number $z=6$ and the dimensionality $d=3$, according to the empirical formula (13), $p_{c}=0.25$. The deviation between the result and the empirical result is caused by a 'surface effect'. As shown in figure 1(c), the two disconnected subsections, $A$ and $B$, turn out to be connected elements, $A^{\prime}$ and $B^{\prime}$, after scaling. Such deviation that is related to the limited formation of the subsection is called the 'surface effect', which is originally from the loss of the resolution in every scaling procedure. Naturally, the more elements a subsection contains, the smaller the loss of resolution will be. So expanding the formation of the subsection can help to reduce the 'surface effect'. In this model, the formation of the subsection is $2 \times 2 \times 2$. If larger formation was employed, such as $3 \times 3 \times 3$, or even $4 \times 4 \times 4$, the results would be better. However, the number of configurations would increase extraordinarily. The number of configurations in a $5 \times 5 \times 5$ formation is estimated to be $3 \times 10^{7} \sim 2^{25}$. To resolve such contradiction, Reynolds and his colleagues [22] introduced a method that combines the RNG approach and Monte Carlo method, called the Monte Carlo renormalization group, to calculate critical parameters with a high degree of accuracy for site percolation by scaling with a large subsection. Brown et al [23] applied this method to 2D 


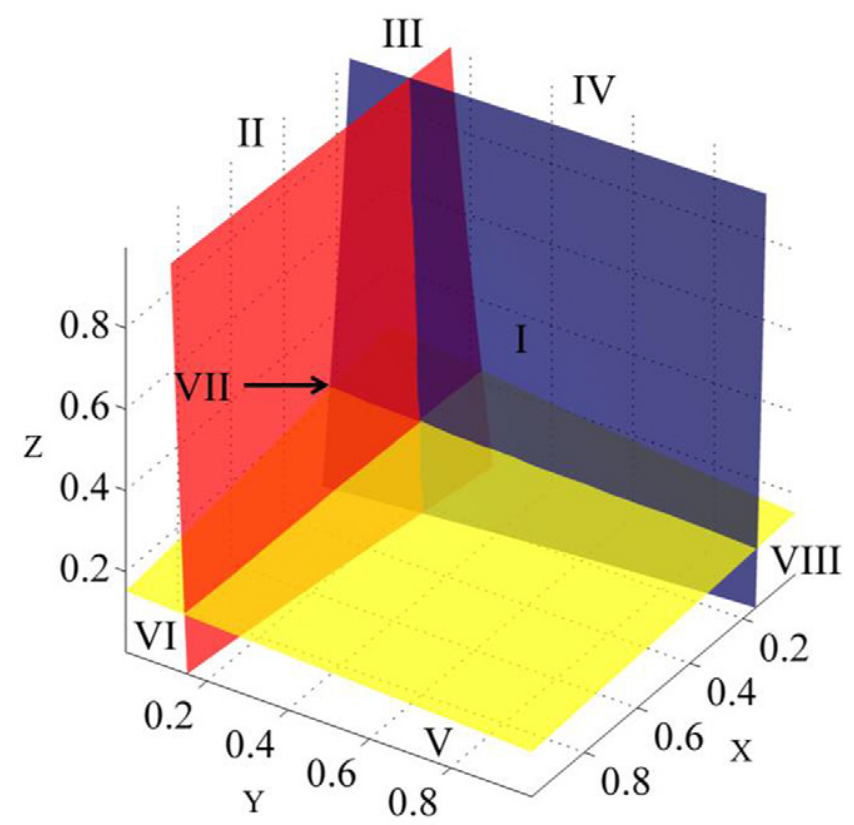

Figure 4. Rock connectivity phase for a $3 \mathrm{D}$ anisotropic crack distribution. The coordinates are the probabilities of the $X, Y$ and $Z$ bonds being in a certain position. The three surfaces, consisting of fixed points, separate the space into eight sections. Each section represents a specific phase as listed in table 2 . The eight corner points, $(0,0,0),(1,0,0),(0,1,0),(1,1,0),(0,0,1),(1,0,1),(0,1,1)$, and $(1,1,1)$ are stable fixed points located at the corner of the eight phase sections, representing eight states when the iteration goes infinitely. The common intersection point of the three surfaces represents the critical points of the 3D isotropic percolation, whose value is 0.2155 .

bond percolation and the renormalized bond probabilities and the values of critical exponents can be determined in this way. Furthermore, Xiong and his co-workers [24] developed this method to investigate the critical behavior of a $3 \mathrm{D}$ random-bond Ising model. However, the Monte Carlo renormalization group method has not been applied in $3 \mathrm{D}$ anisotropic percolation and the results of this method are discrete points in the parameter space, through which a phase diagram is difficult to draw.

A 3D phase diagram for 3D anisotropic percolation was drawn, as shown in figure 4. Similar to the 2D phase diagram, the three surfaces, consisting of fixed points, separate the space into eight sections. Each section represents a specific phase as listed in table 2 . The eight corner points, $(0,0,0),(1,0,0),(0,1,0),(1,1,0),(0,0,1)$, $(1,0,1),(0,1,1)$, and $(1,1,1)$, are stable fixed points located at the corner of the eight phase sections, representing eight states when the iteration goes infinitely, which are the sinks where points in their phase sections flow into. The common intersection point of the three surfaces represents the critical points of the $3 \mathrm{D}$ isotropic percolation, whose value is 0.2155 . The properties of the system on one side of the surfaces are definitely different from the properties of the system on the other side of the surfaces. Such a phenomenon is analogous to a thermodynamic system with distinct phases, which is the reason why the term 'phase diagram' was borrowed from thermodynamics 
Table 2. The phases of the regions in parameter space ${ }^{\mathrm{a}}$.

\begin{tabular}{llll}
\hline & $X$ direction & $Y$ direction & $Z$ direction \\
\hline Region I & $\mathrm{C}$ & $\mathrm{C}$ & $\mathrm{C}$ \\
Region II & $\mathrm{C}$ & $\mathrm{N}$ & $\mathrm{C}$ \\
Region III & $\mathrm{N}$ & $\mathrm{N}$ & $\mathrm{C}$ \\
Region IV & $\mathrm{N}$ & $\mathrm{C}$ & $\mathrm{C}$ \\
Region V & $\mathrm{C}$ & $\mathrm{C}$ & $\mathrm{N}$ \\
Region VI & $\mathrm{C}$ & $\mathrm{N}$ & $\mathrm{N}$ \\
Region VII & $\mathrm{C}$ & $\mathrm{C}$ & $\mathrm{C}$ \\
Region VIII & $\mathrm{N}$ & $\mathrm{C}$ & $\mathrm{N}$ \\
\hline
\end{tabular}

a ' $\mathrm{C}$ ' means the region has connections across the direction. ' $\mathrm{N}$ ' means the region has no connection across the direction.

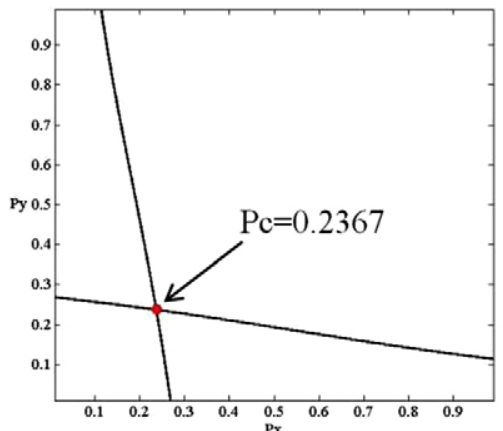

(a)

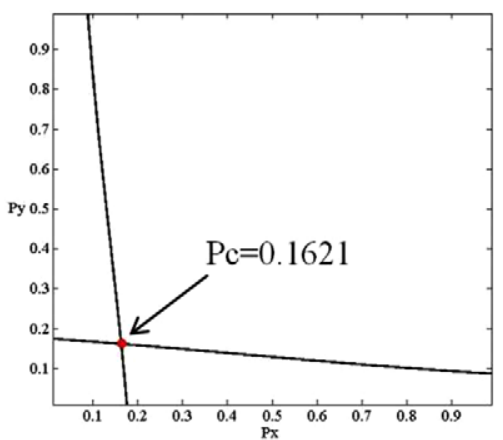

(d)

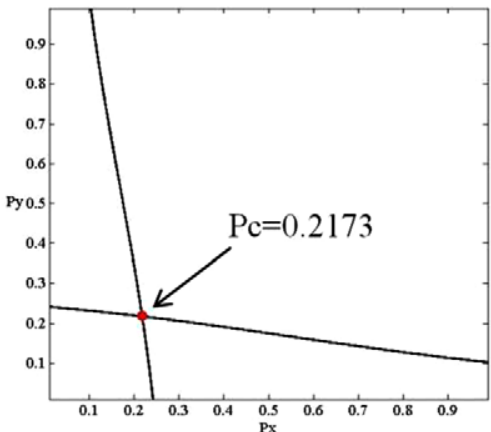

(b)

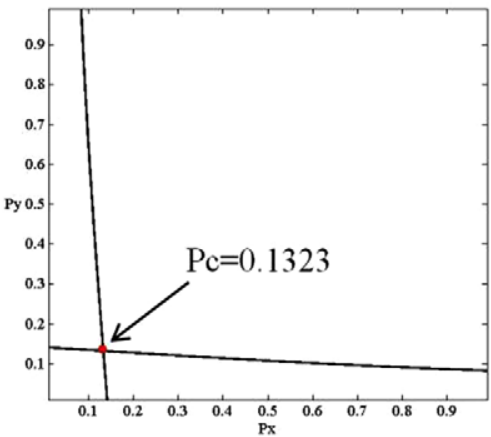

(e)

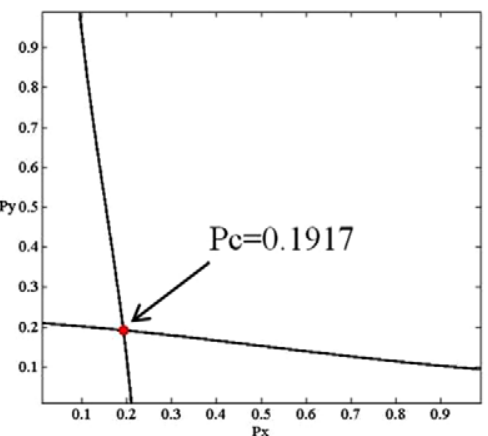

(c)

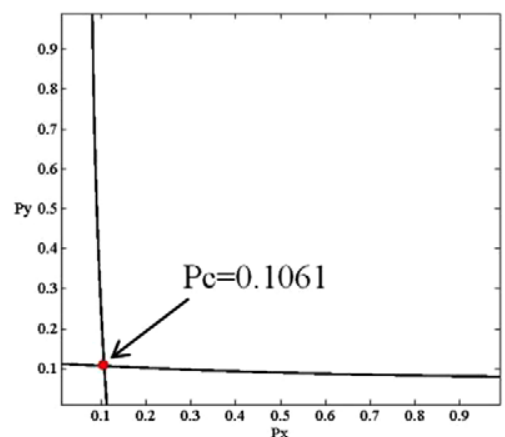

(f)

Figure 5. Slices of figure 5 , when $p_{z}=0, p_{z}=0.2, p_{z}=0.4, p_{z}=0.6, p_{z}=0.8, p_{z}=1$.

[12]. Figure 5 shows slices of the $3 \mathrm{D}$ phase diagram, when $p_{z}=0, p_{z}=0.2, p_{z}=0.4$, $p_{z}=0.6, p_{z}=0.8, p_{z}=1$. When $p_{z}=0$, the phase diagram does not degenerate to the shape of the $2 \mathrm{D}$ phase diagram as figure 3 shows, which can be attributed to the 3D configuration effect for there are more paths to go through in $3 \mathrm{D}$ configuration than in $2 \mathrm{D}$ configuration. As $p_{z}$ increases, the critical points' values decrease, indicating the increase of connecting paths.

The parameters of the phase diagram which are the probabilities of a nearest neighbor connection in rocks in the three orthogonal directions do not merely depend on the crack densities. Actually, after fracking, the fracking pressure $F$, the viscosity $\mu$ and the velocity $V$ of the fracking liquid, and the porosity $n$ and surface energy $\gamma$ of the rock could also influence the probabilities. Additionally, fracking processes are associated 


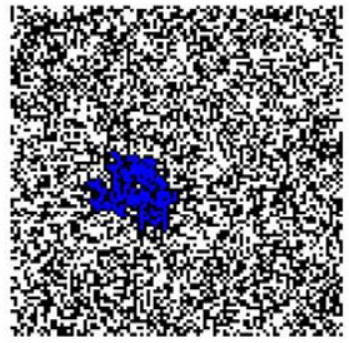

(a)

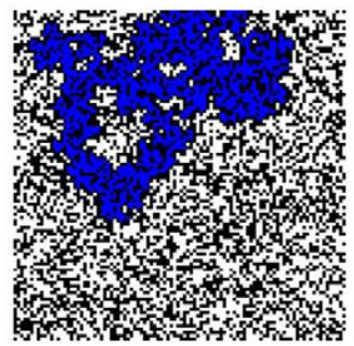

(c)

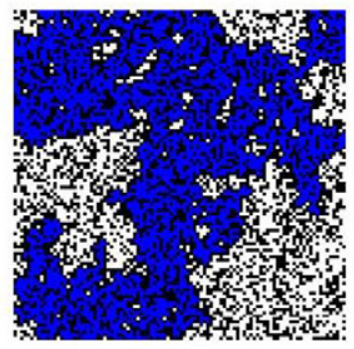

(e)

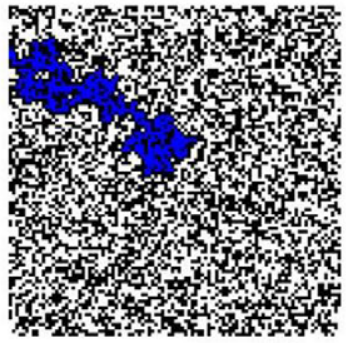

(b)

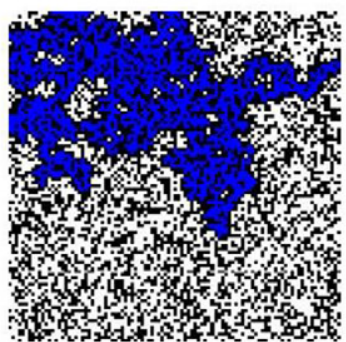

(d)

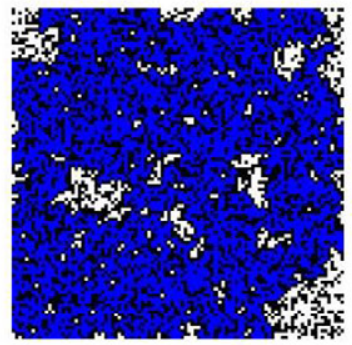

(f)

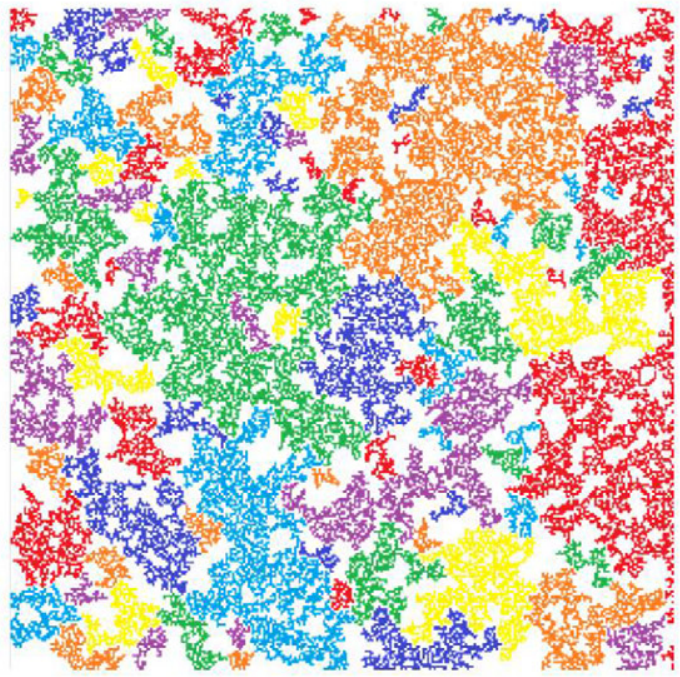

(g)

Figure 6. (a)-(f) The largest connected clusters of $2 \mathrm{D}$ percolation with different values of $p$ given as $0.51,0.53,0.55,0.57,0.59$, and 0.61 . Obviously, the largest connected cluster expands with the increase of the value of $p$. (g) A simulation of $2 \mathrm{D}$ percolation with $p=0.5$ rendered with different colors which represent the different connected clusters. 


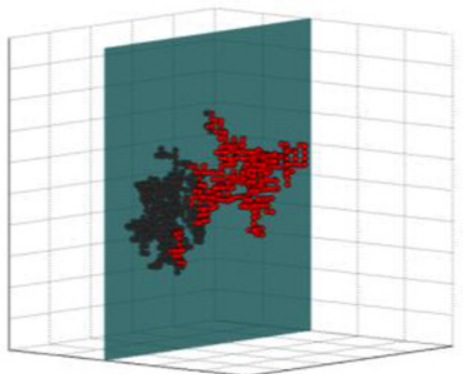

(a)

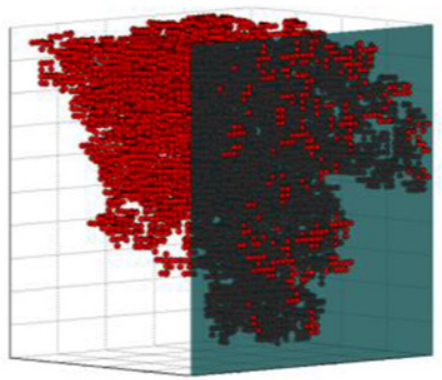

(c)

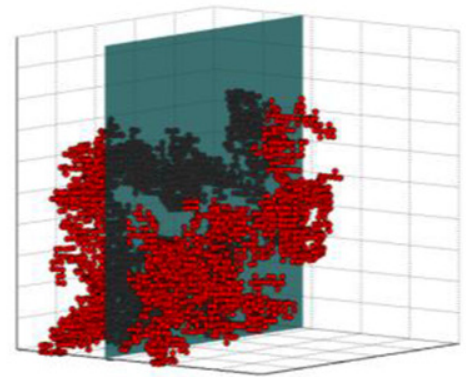

(b)

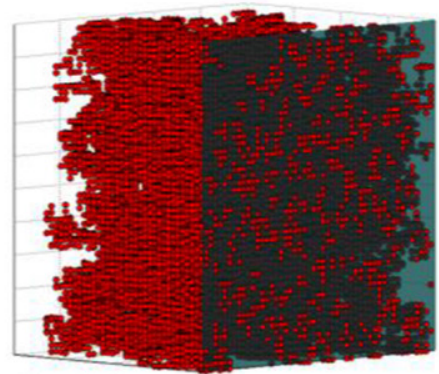

(d)

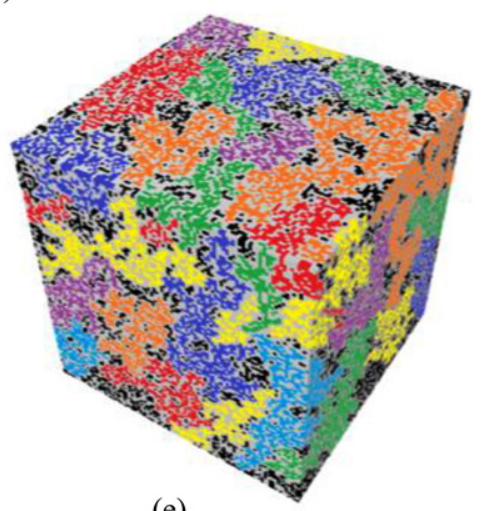

(e)

Figure 7. (a)-(d) The largest connected clusters in $3 \mathrm{D}$ percolation with different values of $p$ given as $0.29,0.31,0.33$ and 0.35 . (e) A simulation of $3 \mathrm{D}$ percolation with $p=0.3$ rendered with different colors which represent the different connected clusters.

with the creation of extensive artificial fractures [25], so the probabilities of a nearest neighbor connection should be the results of the combined effects from existing (natural) fractures, potential cracks that can be connective under specific pressure, and newly created fractures. Thus equation (8) can be modified as

$$
p_{x}=f\left(\frac{N_{x} L^{2}}{S}, F, \gamma, n, V, \mu, d\right),
$$

where $N_{x} L^{2} / S$ is the crack density in the $X$ direction and $d$ is the characteristic scale of the holes in the rock. According to dimensional analysis, the variables in the above equation were combined and two dimensionless numbers were found as follows: 
Microcrack connectivity in rocks

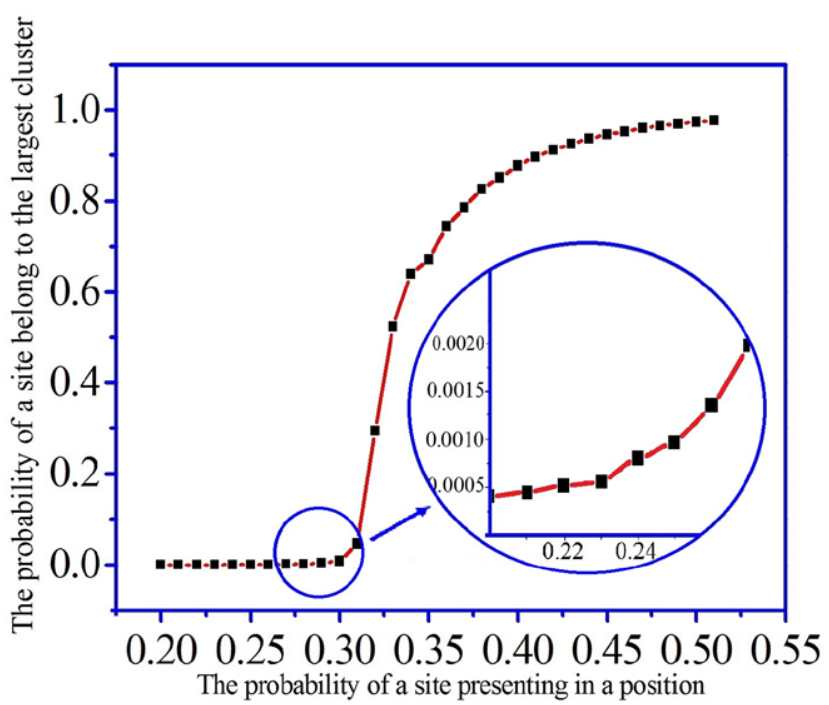

Figure 8. The probabilities of a site belonging to the largest clusters in 3D percolation with different values of $p$.

$$
p_{x}=f\left(\frac{N_{x} L^{2}}{S}, \frac{F d}{\mu V+a \gamma}\right)
$$

where $F d /(\mu V+a \gamma)$ represents the competition among the fracking pressure, the viscous forces of the fracking liquid and the bond force in the rock while $a$ denotes an undefined coefficient. It reflects the coupled effect caused by potential cracks and newly created cracks. It indicates the function in equation (15) which can be used to predict how well the fracking strengthens the permeability of a rock.

A series of simulations for the isotropic site percolation has been performed. Figures 6 and 7 show the images of the largest connected clusters with different values of $p$ in 2D and $3 \mathrm{D}$ percolation, respectively. Interestingly, the size of the connected clusters grows rapidly when $p$ is given bigger than the percolation threshold where $p_{c}$ is about 0.5 in $2 \mathrm{D}$ and $p_{c}$ is about 0.25 in $3 \mathrm{D}$. And the statistic of the probabilities of a site belonging to the largest clusters with different values of $p$ in $3 \mathrm{D}$ is shown in figure 8 , which indicates that the size of the largest clusters starts to grow when $p \approx 0.23$ which is between the model's result, $p_{c}$ of about 0.2155 , and the empirical value, $p_{c}$ of about 0.25 .

\section{Conclusions}

A 3D anisotropic percolation model has been modified. The reduced results to isotropic percolation are in good agreement with a series of previous calculations for the percolation threshold $p_{c}$ and the correlation length exponent $v$. The results of the anisotropic percolation are presented as phase diagrams which predict the permeability of the rock according to the crack density. The results have been demonstrated to be more suitable for the needs of engineering than Nakanishi et al.'s work for the better description of critical behavior compared with the results of simulation. 
Table B1. The approximations of the roots of Nakanishi et al's equation

\begin{tabular}{ll}
\hline Number & The approximations of the roots \\
\hline 1 & 0 \\
2 & 1 \\
3 & 3.1117464899844339060710096233615 \\
4 & 0.047678810565100527628670623573103 \\
5 & -0.12264803040771493034409074548009 \\
6 & 1.8840193157902460234304616802865 \\
7 & $-0.49682424082449828141315094099147+0.41394204141653873165319952650177 \mathrm{i}$ \\
8 & $-0.49682424082449828141315094099147-0.41394204141653873165319952650177 \mathrm{i}$ \\
9 & $0.89659469041575471145277943889664+0.2021653569761044759885942081425 \mathrm{i}$ \\
10 & $0.89659469041575471145277943889664-0.2021653569761044759885942081425 \mathrm{i}$ \\
11 & $1.2552158728273261911827305266089+0.11109722171342945991171425430694 \mathrm{i}$ \\
12 & $1.2552158728273261911827305266089-0.11109722171342945991171425430694 \mathrm{i}$ \\
\hline
\end{tabular}

\section{Appendix A}

In Nakanishi et al's work [20], the percolation threshold in the $2 \times 2 \times 2$ subsection was not calculated. The isotropic percolation threshold could be easily derived from the scaling relations. The scaling relations derived by [20] are shown as follows:

$$
\begin{aligned}
a^{\prime}= & a^{8}+8 a^{7}(1-a)+28 a^{6}(1-a)^{2}+56 a^{5}(1-a)^{3} \\
& +2 a^{4}(1-a)^{4}\left(68 C_{22}+136 C_{21}+136 C_{12}+66 C_{20}+264 C_{11}+66 C_{02}\right. \\
& \left.+124 C_{10}+124 C_{01}+54 C_{00}\right)+8 a^{3}(1-a)^{5}\left(48 C_{22}+96 C_{21}+96 C_{12}\right. \\
& \left.+40 C_{20}+168 C_{11}+40 C_{02}+64 C_{10}+64 C_{01}+24 C_{00}\right)+4 a^{2}(1-a)^{6} \\
& \times\left(16 C_{22}+32 C_{21}+32 C_{12}+8 C_{20}+40 C_{11}+8 C_{02}\right. \\
& \left.+12 C_{10}+12 C_{01}+4 C_{00}\right) \\
\equiv & f(a, b, c), \\
b^{\prime}= & f(b, c, a), \\
c^{\prime}= & f(c, a, b),
\end{aligned}
$$

where $C_{i j}$ denotes $b^{i}(1-b)^{2-i} c^{j}(1-c)^{2-j}$ and $a, b, c$ represent the probability of an $X$, $Y, Z$ bond being in a certain position, respectively.

In isotropic percolation, $a=b=c$. So the isotropic threshold should be one of the roots of the following equation

$$
a=f(a, a, a) .
$$

The approximations of the roots are shown in table B1. There is only one root whose value is a real number and between 0 and 1 , which is about 0.0477 .

\section{Appendix B}

In $2 \mathrm{D}$ isotropic percolation where $\mu=p$, the procedure of scaling the square lattice can be written as

$$
p^{\prime}=R(p)=2 p^{2} q^{3}+8 p^{3} q^{2}+5 p^{4} q+p^{5},
$$




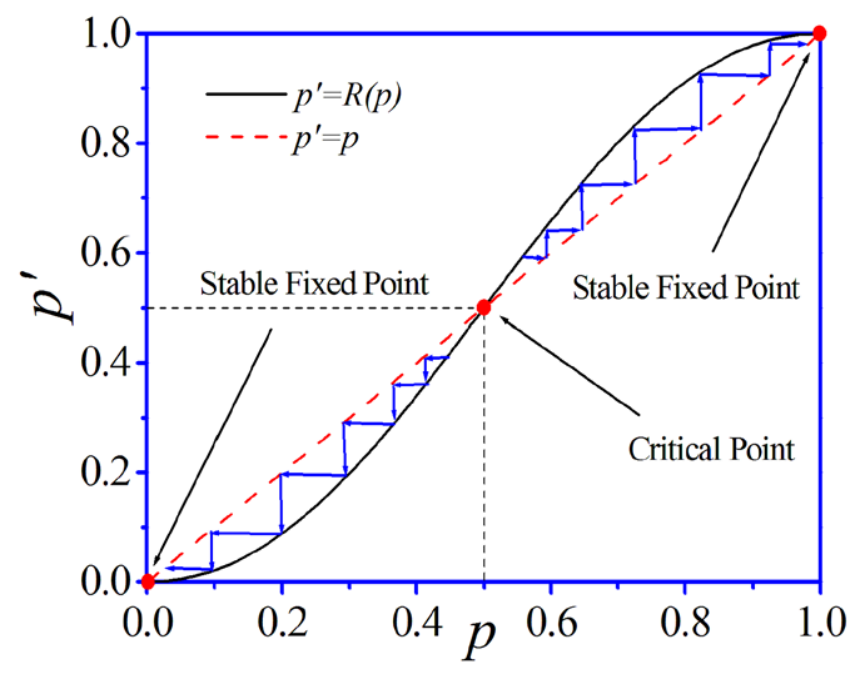

Figure B1. An illustration of the scaling procedure in a mathematical way. The black curve represents the iteration function and the arrows denote the change of $p^{\prime}$ in every scaling procedure.

where $p$ is the probability with which a bond presents, $q=1-p$, and $p^{\prime}$ is the probability in the large-scale subsection. The curve of the iteration function is shown in figure $\mathrm{B} 1$, where $p=0$ and $p=1$ are stable fixed points attracting another near them during the scaling procedure and $p=0.5$ is an unstable fixed or critical point $p_{c}$ that acts like a boundary separating the drainage areas that feed the stable fixed point. For an infinite network, if its bonds present with a probability which is less than $p_{c}$, it will have zero probability of being connected, while if its bonds present with a probability greater than $p_{c}$, it will have one probability of being connected.

\section{Acknowledgments}

This work was jointly supported by the National Natural Science Foundation of China (Grant Nos. U1562105 and 11372313) and CAS Interdisciplinary Innovation Team Project.

\section{References}

[1] Norris J Q, Turcotte D L and Rundle J B 2014 Loopless nontrapping invasion-percolation model for fracking Phys. Rev. E 89022119

[2] Bažant Z P, Salviato M, Chau V T, Viswanathan H and Zubelewicz A 2014 Why fracking works J. Appl. Mech. 81101010

[3] Olson J E, Laubach S E and Lander R H 2009 Natural fracture characterization in tight gas sandstones: integrating mechanics and diagenesis AAPG Bull. 931535

[4] Gale J F W and Holder J 2010 Natural fractures in some US shales and their importance for gas production Geological Society, London, Petroleum Geology Conf. Series vol 7 pp 1131-40

[5] Hammersley J M 1957 Percolation processes Math. Proc. Camb. 53642

[6] Norris J Q, Turcotte D L and Rundle J B 2015 Anisotropy in fracking: a percolation model for observed microseismicity Pure Appl. Geophys. 1727

[7] Young A P and Stinchcombe R B 1975 A renormalization group theory for percolation problems J. Phys. C: Solid State Phys. 81535 
[8] Harris A B, Lubensky T C, Holcomb W K and Dasgupta C 1975 Renormalization group approach to percolation problems Phys. Rev. Lett. 35327

[9] Kasteleyn C M and Fortuin P W 1972 Random-cluster model: introduction and relation to other models Physica $\mathbf{5 7} 536$

[10] Stinchcombe R B and Watson B P 1976 Renormalization group approach for percolation conductivity J. Phys. C: Solid State Phys. 93221

[11] Reynolds P S, Klein W and Stanley H E 1977 A real space renormalization group for site and bond percolation J. Phys. C: Solid State Phys. 101167

[12] Madden T R 1983 Microcrack connectivity in rocks: a renormalization group approach to the critical phenomena of conduction and failure in crystalline rock J. Geophys. Res. $8 \mathbf{8} 585$

[13] Wilkinson D and Willemsen J F 1983 Invasion percolation: a new form of percolation theory J. Phys. A: Math. Gen. 163365

[14] Furuberg L, Feder J, Aharony A and Jossang T 1988 Dynamics of invasion percolation Phys. Rev. Lett. 612117

[15] Maslov S 1995 Time directed avalanches in invasion models Phys. Rev. Lett. 74562

[16] Paczuski M, Maslov S and Bak P 1996 Avalanche dynamics in evolution, growth, and depinning models Phys. Rev. E 53414

[17] Ebrahimi F 2010 Invasion percolation: a computational algorithm for complex phenomena Comput. Sci. Eng. 1284

[18] Porto M, Havlin S, Schwarzer S and Bunde A 1997 Optimal path in strong disorder and shortest path in invasion percolation with trapping Phys. Rev. Lett. 794060

[19] Knackstedt M A, Sahimi M and Sheppard A P 2002 Nonuniversality of invasion percolation in two-dimensional systems Phys. Rev. E $\mathbf{6 5} 035101$

[20] Nakanishi H, Reynolds P J and Redner S 1981 Anisotropic bond percolation by position-space renormalisation group J. Phys. A: Math. Gen. 14855

[21] Lorenz C D and Ziff R M 1998 Precise determination of the bond percolation thresholds and finite-size scaling corrections for the sc, fcc, and bcc lattices Phys. Rev. E 57230

[22] Reynolds P J, Stanley H E and Klein W 1980 Large-cell Monte Carlo renormalization group for percolation Phys. Rev. B 211223

[23] Brown A, Edelman A, Rocks J, Coniglio A and Swendsen R H 2013 Monte Carlo renormalization-group analysis of percolation Phys. Rev. E 88043307

[24] Xiong W, Zhong F, Yuan W and Fan S 2010 Critical behavior of a three-dimensional random-bond Ising model using finite-time scaling with extensive Monte Carlo renormalization-group method Phys. Rev. E 81051132

[25] Li D, Wang F, Yang Z and Zhao Y 2014 How to identify dislocations in molecular dynamics simulations Sci. China-Phys. Mech. Astron. 572184 